Int. J. Curr. Res. Med. Sci. (2017). 3(7): 56-61

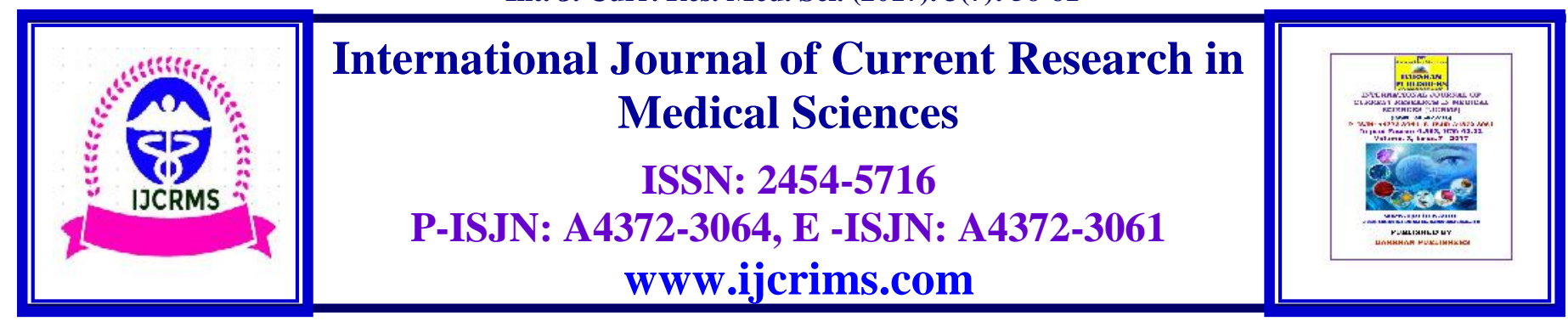

Case Report

Volume 3, Issue 7 -2017

DOI: http://dx.doi.org/10.22192/ijcrms.2017.03.07.009

\title{
Primary Rifampicin Drug Resistant Disseminated Extrapulmonary Tuberculosis
}

\author{
*Srijna Rana, * Rajwinder Kaur **NC Kajal , *Nishanth PS, ***N.S.Neki \\ *Junior Resident, **Professor, Chest \& TB Dept. Govt. Medical College, Amritsar, \\ India, 143001 \\ ***Professor of Medicine, Govt. Medical College, Amritsar, India \\ Corresponding Author: Srijna Rana \\ E-mail: srijnarana@yahoo.com
}

\begin{abstract}
Disseminated tuberculosis (TB) refers to involvement of two or more non-contiguous sites. It is a common mode of presentation of tuberculosis in patients both with and without HIV/AIDS in India. However, the presence of primary drug resistance in disseminated tuberculosis involving only the extrapulmonary sites in an immunocompetent adult is rare. Extrapulmonary TB accounts for approximately 15\% of the TB cases among immunocompetent hosts and for $50 \%-70 \%$ of cases of TB occurring in immunocompromised individuals, especially in persons with HIV. Rifampicinresistant tuberculosis is caused by bacteria that do not respond to Rifampicin, one of the most powerful anti-TB medicines. These patients require MDR-TB treatment. Here we present a case of a 32 year old male painter with primary Rifampicin resistant disseminated tuberculosis in the form of cold abcess, tuberculous spondylitis, epidydmitis and scrofuloderma.
\end{abstract}

Keywords: Disseminated tuberculosis, Extrapulmonary tuberculosis, Primary resistance

\section{Introduction}

In 2015, there were an estimated 480000 new cases of multidrug-resistant TB (MDR-TB) and an additional 100000 people with Rifampicinresistant TB (RR-TB) who were also newly eligible for drug resistant-TB treatment. Drug resistance surveillance data show that $3.9 \%$ of new and $21 \%$ of previously treated TB cases were estimated to have had Rifampicin or multidrugresistant tuberculosis (MDR/RR-TB) in 2015. As in 2014, MDR-TB accounts for $3.3 \%$ of new TB cases. MDR/RR-TB caused 250000 deaths in 2015. Most cases and deaths occurred in Asia.[1].
As there is an increase in the number of pulmonary MDR-TB cases throughout the world, it is predicted that extra-pulmonary presentation would also become higher than before in the coming future. Drug resistance has been described as either primary or acquired. Primary drug resistance refers to resistance of strains segregated from patients who have not formerly received TB treatment. Acquired drug resistance expressed TB isolated from patients who presently are getting or until that time have received anti-TB drug treatment for at least 1 month. Primary drug 
resistance is understood to be caused by the spread of drug-resistant strain. Primary Rifampicin resistant extrapulmonary cases involving multiple extrapulmonary sites in an immmunocompetent individual is quite rare in literature $[2,3,4]$

\section{Case Report}

A 32 year old male painter with no history of anti tuberculosis treatment presented with insidious onset of multiple non tender subcutaneous swellings on various parts of the body with the largest one in the para spinal lumbar region for last 9 months. (See figure 1). Other areas were postauricular and paracervical region. Over the next few months the patient started experiencing vague backache. Other presenting complaints were appearance of multiple skin lesions with spontaneous rupture and recurrence at various parts of the body and intermittent fever, painful scrotal swelling and loss of appetite since 2 months. There was no history of cough, expectoration, haemoptysis, or breathlessness. Thick caseous cream colored pus was aspirated from the abscess and sent for culture and CBNAAT . There was no growth of any pyogenic organism after 48 hours of the incubation, but acid fast bacilli (AFB) were seen in Z-N stain and it came out to be tubercular with Rifampicin resistant on CBNAAT. There was no history of contact and no history of any previous treatment of tuberculosis. Chest examination showed no clinical abnormality. The examination of cardiovascular, abdominal and neurological systems was unremarkable. There was no clinical evidence of any immunosupression.

On examination, his vital signs were stable and pallor was present. There was no significant lymphadenopathy. A swelling of $7 \times 6 \mathrm{~cm}$ was present over the para spinal lumbar region with ill-defined borders (figure 1), which was nontender and soft in consistency. The skin over the swelling was normal. The swelling was fluctuant and non-transluminant. There was no palpable bony defect underneath the swelling. There were multiple skin lesions present over the back of trunk, and in cervical and axillary regions.
Blood investigations revealed haemoglobin of $11.1 \mathrm{~g} / \mathrm{dl}$, total leucocyte count 9500/ 1 with differential counts (neutrophils 77\%, lymphocytes $23 \%$ and eosinophils $0 \%$ ) and ESR $35 \mathrm{~mm}$ and RBS of $96 \mathrm{mg} / \mathrm{dl}$. He had normal liver and renal function tests. Serological evidence for HIV infection was negative. His X-ray chest was unremarkable.

MRI spine (figure 2) was done to find the cause of backache which showed involvement of cervical, dorsal and lumbar vertebrae ; edematous changes and collection in epidural space at D4-D6 suggestive of Pott's spine. USG scrotum was suggestive of epidydmitis and culture of skin biopsy specimens indicated Mycobacterium tuberculosis with histopathology revealing the presence of characteristic tubercular granulomas with epithelioid cells. Pus discharge from the lesions was stained for AFB.

The patient was started on DOTS CAT IV under Revised National TB Control Programme.He responded to treatment with regression of symptoms i.e. healing of the skin lesions and decrease in the scrotal swelling. Thus he was diagnosed as a case of primary rifampicinresistant disseminated extrapulmonary tuberculosis.

\section{Discussion}

Tuberculosis (TB) is an infectious disease caused by airborne transmission by the acid fast bacillus Mycobacterium tuberculosis and is an important cause of death worldwide [5]. Since, 1990 the drug resistant TB has become a major public health problem [6] . Although pulmonary form of drug resistant $-\mathrm{TB}$ is widely reported but extrapulmonary forms are reported rarely. And resistance being primary in extrapulmonary $\mathrm{TB}$ cases is in itself an uncommon presentation, even in the immunocompromised patients, and it requires a great deal of suspicion to diagnose such a case [7]. There is scanty information regarding extra-pulmonary primary drug resistant-TB in the literature [8]. 
Int. J. Curr. Res. Med. Sci. (2017). 3(7): 56-61

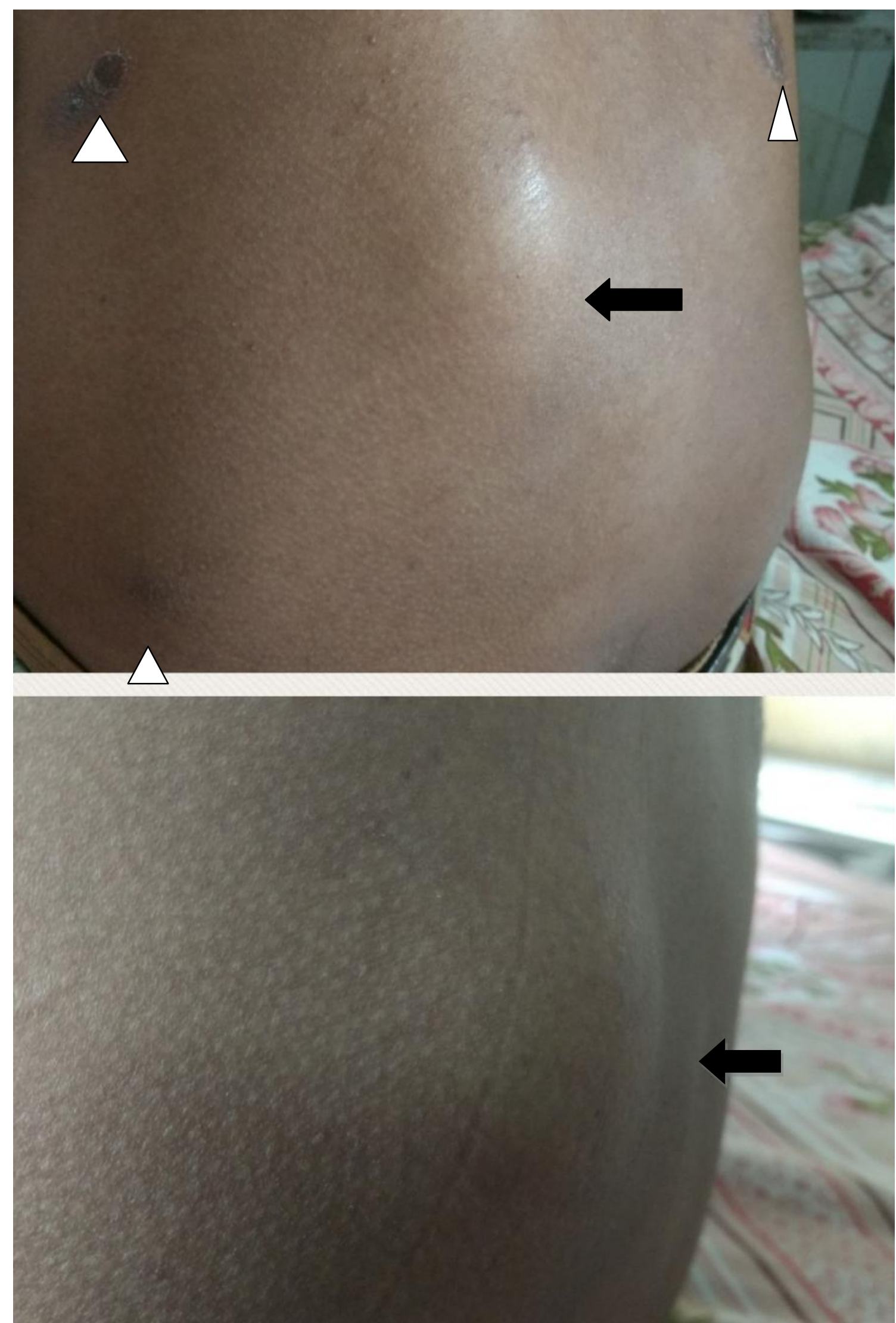

Figure 1- Cold abscess in lumbar region (arrrow) and Healed stages of skin lesions (arrowhead) 
Int. J. Curr. Res. Med. Sci. (2017). 3(7): 56-61

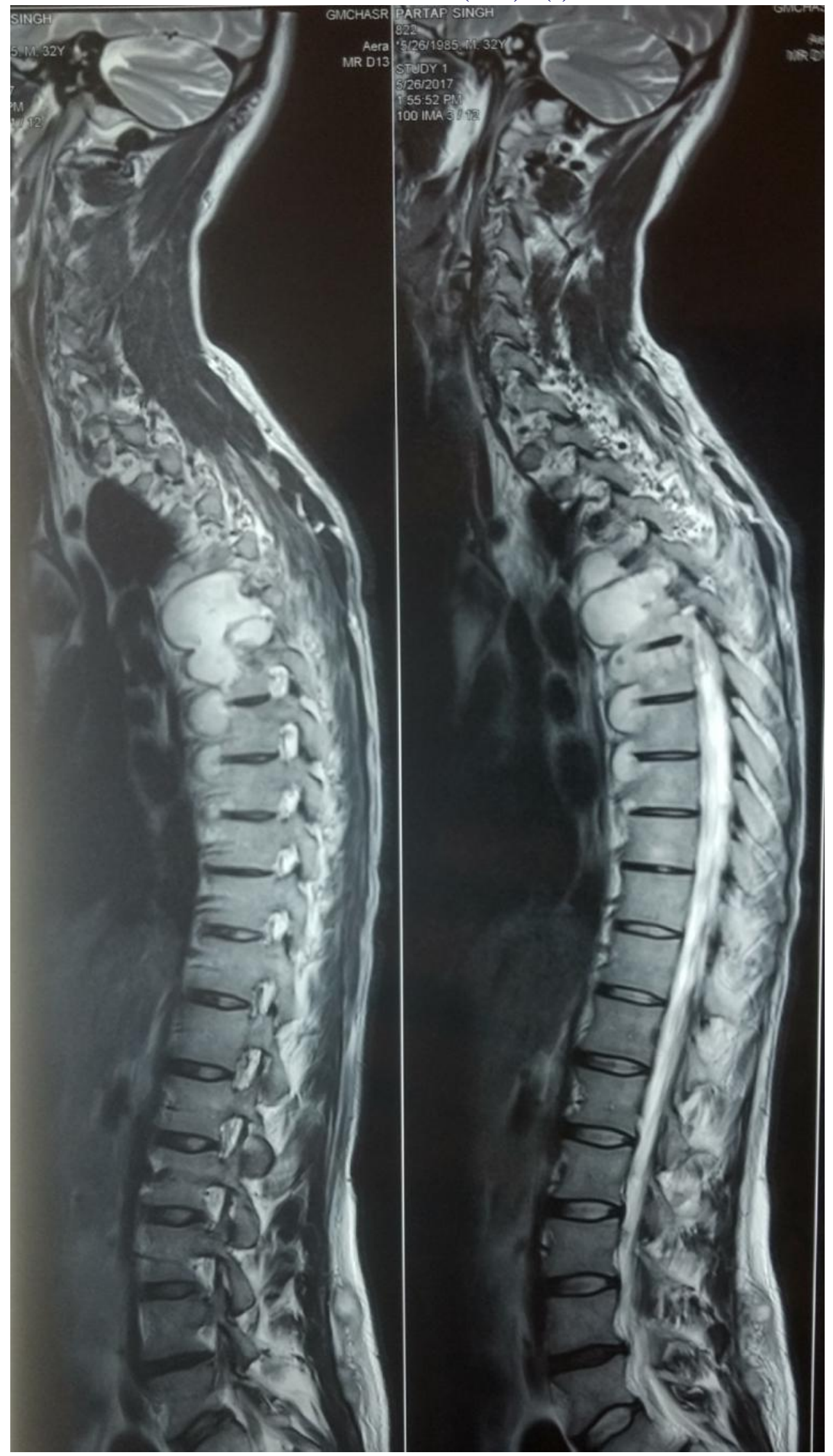

Figure 2 - MRI spine indicating tuberculous spondylitis 
Int. J. Curr. Res. Med. Sci. (2017). 3(7): 56-61

A substantial proportion of patients with multidrug-resistant tuberculosis might have transmitted drug resistance (ie, present before treatment begins), and drug resistance is therefore often the cause of treatment failure, not the result. Several studies have illustrated how, as multidrug-resistant tuberculosis epidemics take hold in populations, most multidrug-resistant tuberculosis cases can shift from being acquired during treatment to primarily transmitted. At least one modeling analysis suggests that this transition has already taken place in many countries throughout the world [9]. The drug resistant-TB develops due to spontaneous mutations in the genes of the bacilli. The drug resistant-TB is basically a man-made problem, as a result of improper or poorly administered treatment [10].

Tubercular cold abscess is also a form of extrapulmonary TB. An abscess is a collection of pus within the body. A "cold abscess" is cold because it is not accompanied by the classical signs of inflammation. Cold abscesses are almost always a sequelae of tubercular infection anywhere in the body. It usually occurs in association with vertebral TB after hematogenous spread and develops as an exudative lesion due to hypersensitivity reaction to mycobacterial TB.[11]. Skeletal TB comprises about $10 \%$ of the extrapulmonary cases. TB spondylitis accounts for $50 \%$ of the skeletal TB cases. Hence, in all, osteoarticular TB represents $1-2 \%$ and TB spondylitis represents $0.5-1 \%$ of all TB cases.[12] Contrary to the previously reported drug resistance, presentation was different in our case. Here the patient neither had history of tuberculosis in the past or any contact history indicating primary resistance. Primary Drug resistance to Mycobacterium tuberculosis results from unplanned and accidental mutations in the bacterial chromosome that results in condensed vulnerability to unambiguous agents. There is no confirmation that acquired genes or plasmids play a role in the coming out of antimicrobial resistance in mycobacterium. The prevalence of MDR-TB is increasing in number in recent years because of increased awareness of the disease, increased access to culture and drug sensitivity testing, and earlier suspicion of MDR-TB cases among previously treated patients. So, it is expected that MDR-TB affecting extrapulmonary sites will increase in the near future. [7] Hence, there is an urgent need for increasing awareness of the physicians to such presentation.

\section{Conclusion}

This rare case is reported here with an aim to create awareness about the possibility of drug resistant-TB in extrapulmonary sites. It also emphasizes the need for rapid diagnosis by genotypic/molecular drug sensitivity testing (genexpert or line probe assay) to detect drug resistant-TB, particularly when extrapulmonary site yields mycobacteria in cases having no history of antitubercular therapy.

\section{Source of funding: Nil}

Conflict of interest: None declared

\section{References}

1. WHO. Global tuberculosis report 2016. Geneva: World Health Organization, 2016

2. Manu MK, Mohapatra AK, Magazine R. Extrapulmonary multidrug resistant tuberculosis presenting as chest wall abscessa rare case report. Indian J Tuberc 2013; 60:121-3. New Delhi.

3. Sarkar S, Maity GN, Mukhopadhyay KK, Acharyya B, Ghoshal AG. Primary multidrug resistant tuberculosis. Lung India 2007; 24:979.

4. Das S K, Das A, Gangopadhyay A, Sinha A K. Primary disseminated extrapulmonary multidrug resistant tuberculosis. Indian J Med Microbiol 2012;30:364-6

5. Yadav S, Rawal G. Tubercular Nodular Episcleritis: A Case Report. [1]J Clin Diagn Res. 2015;9(8):ND01-02.

6. Pablos-Méndez A, Raviglione MC, Laszlo A, Binkin N, Rieder HL, Bustreo F, et [2]al. Global surveillance for antituberculosis-drug resistance, 1994-1997. World Health Organization-International Union against Tuberculosis and Lung Disease Working Group on Anti-Tuberculosis Drug Resistance Surveillance. $N$ Engl J Med. 1998;338(23):1641-9. pmid: 9614254. 
7. Rawat J, Sindhwani G, Dua R. Primary multidrug resistant tubercular lymphadenitis in an HIV infected patient. Indian $J$ Tuberc. 2009;56:157-59.

8. Sharma SK, Mohan A. Multi-drug resistant extra-pulmonary tuberculosis in a HIV negative patient. Indian J Tuberc. 2004;51:4346.

9. Kendall EA, Fofana MO, Dowdy DW. Burden of transmitted multidrug resistance in epidemics of tuberculosis: a transmission modelling analysis. Lancet Respir Med 2015; 3: $963-72$

10. Mittal N, Bansal P. Multidrug resistant extrapulmonary tuberculosis - three case reports and review of literature. Intern Med Inside. 2014;2:2.

11. Bhan S, Nag HL. Skeletal tuberculosis. In: Tuberculosis, Sharma SK (Ed.). 2nd ed. New Delhi: Jaypee, 2009. pp 243.

12. Polley P, Dunn R. Noncontiguous spinal tuberculosis: Incidence and management. Eur Spine J. 2009;18:1096-101

\begin{tabular}{|c|l|}
\hline \multicolumn{2}{|c|}{ Access this Article in Online } \\
\hline & Website: \\
\hline & www.ijcrims.com \\
\cline { 2 - 2 } & Subject: \\
\hline Quick Response Code & \\
\hline
\end{tabular}

How to cite this article:

Srijna Rana, Rajwinder Kaur, NC Kajal, Nishanth PS, N.S.Neki. (2017). Primary Rifampicin Drug Resistant Disseminated Extrapulmonary Tuberculosis. Int. J. Curr. Res. Med. Sci. 3(7): 56-61.

DOI: http://dx.doi.org/10.22192/ijcrms.2017.03.07.009 